Article

\title{
vIS: An Immersive Virtual Storytelling System for Vocational Training
}

\author{
Sanika Doolani *(D), Luke Owens, Callen Wessels and Fillia Makedon* \\ Department of Computer Science and Engineering, The University of Texas at Arlington, Arlington, TX 76019, USA; \\ luke.owens@mavs.uta.edu (L.O.); callen.wessels@mavs.uta.edu (C.W.) \\ * Correspondence: sanikasunil.gupta@mavs.uta.edu (S.D.); makedon@uta.edu (F.M.)
}

Received: 20 October 2020; Accepted: 11 November 2020; Published: 17 November 2020

check for updates

\begin{abstract}
Storytelling has been established as a proven method to effectively communicate and assist in knowledge transfer. In recent years, there has been growing interest in improving the training and learning domain by using advanced technology such as Virtual Reality (VR). However, a gap exists between storytelling and VR, and it is as yet unclear how they can be combined to form an effective system that not only maintains the level of engagement and immersion provided by VR technology but also provides the core strengths of storytelling. In this paper, we present vIS, a Vocational Immersive Storytelling system, which bridges the gap between storytelling and VR. vIS focuses on vocational training, in which users are trained on how to use a mechanical micrometer by employing a creative fictional story embedded inside a virtual manufacturing plant's workplace. For the evaluation, a two-phase user study with 30 participants was conducted to measure the system's effectiveness and improvements in long-term training, as well as to examine user experience against traditional methods of training-2D videos and textual manuals. The results indicate that the user's ability to retain their training after seven days was nearly equal for vIS and the 2D video-based technique and was considerably higher than the text-based technique.
\end{abstract}

Keywords: virtual reality; storytelling; vocational training; usability testing

\section{Introduction}

Some of the most important factors behind a country's rising poverty and unemployment have been tied to the population's skill level and its ability to gain new skills in a changing technological landscape. The first response of policymakers to this has been to launch new vocational training initiatives to upgrade the skill level of the population, especially the youth [1,2]. Employers invest an average of $\$ 3,000,000$ per year into upgrading the skills of their workforce [3], mostly by assigning mentors to oversee training. Thus, the need for a cheap training replacement which offers the same level of training has recently become a key area of interest.

To tackle this problem, researchers have worked extensively on producing innovative computer-based techniques to train and prepare workers for various positions in industry. We have seen a great increase in Human-Robot-Interaction (HRI)-based applications [4-7] and Augmented and Virtual Reality (AR/VR) applications [8,9] targeted towards teaching new skills, whether vocational or otherwise.

While HRI and AR-based vocational training require a user to be physically present in the environment for which the training is curated, Virtual Reality on the other hand offers an entirely different experience in which a user can be completely immersed in a virtual simulation of the same environment. This immersive 
capability of Virtual Reality systems have been found to enhance engagement [10-12] and cognitive capabilities, such as recall [13-15]. While such applications do succeed in imparting new skills [16], they fail in terms of providing the social and emotional interaction experience that comes from undertaking the same training in person and on-site.

From a sociological viewpoint, there has been a widespread and growing need for and acceptance of interactive digital storytelling technologies $[17,18]$. At its core, digital storytelling allows computer users to become creative storytellers through the traditional processes of selecting a topic, conducting research, writing a script and developing an interesting story. A story can be curated in the form of a personal narrative focusing on educational content.

While much research has been conducted on using Virtual Reality-based training systems, none of them has explored the advantages of adding storytelling content to such systems to measure the impact on learning outcomes, user engagement and long-term recall. In this work, we present the Vocational Immersive Storytelling (vIS) system, a novel immersive virtual storytelling system for vocational training. We investigate the effectiveness of adding fictional story content inside an immersive virtual reality system that is designed to train users on how to use a micrometer-an advanced tool whose demand for skilled knowledge exceeds educational training [19]. We evaluate our system's capability to provide a training that is suitable for long-term recall in comparison to traditional methods of vocational training, text-based and video tutorial-based learning by conducting a two-phase user study of 30 participants spaced seven days apart. We also present the usability and user engagement metrics of our vIS system against the traditional methods of training.

Our analysis shows that the participants of our vIS system performed slightly better than 2D video-tutorial participants, but outperformed the participants of a text-based training method in terms of long-term retention and recall regarding the use of a micrometer. Data collected from post-training questionnaires show a high system usability score (SUS) [20] of 76 for our vIS system, which indicates a high degree of satisfaction in using our system compared to other traditional methods.

In the following sections, we present the relevant work in this context, describe the design of vIS in detail along with the design decisions behind the story content and then report the insights of our user study, which is followed by a discussion of our findings.

\section{Related Work}

\subsection{Vocational Training in the Workplace}

There have been many studies regarding the inadequacies and difficulties of vocational training. Billet et al. [21] discusses training in the workplace wherein training benefits the student by providing authentic activities, engaging tasks and access to experts and other workers. However, there are also many limitations of workplace training, including undesirable knowledge, the reluctance of experts and knowledge which is difficult to acquire [21]. Smith et al. explained that, while many students prefer "on-the-job" training, many workplaces are not equipped to provide the training necessary to facilitate readiness [22]. Other works include research into language acquisition [16], which focused more on learning a second-language using Virtual Reality. Bruijn et al. assessed various vocational courses in the Netherlands and found that, in a survey of course coordinators, stronger methods of education were "[strongly] desired" and that students did not feel they were gaining necessary experience [23]. In an effort to improve vocational training while still maintaining the on-the-job experience, our vIS system places the trainee in a workplace environment that is controlled, engaging and has many of the benefits of real-world workplace training such as having authentic activities, engaging tasks and context-based experience. The ability to script the training sequence eliminates the risk of undesirable or unclear information and does not rely on workplace employees' willingness to teach. 


\subsection{Access to Vocational Training}

Virtual Reality has been utilized around the world to increase accessibility to vocational training [24,25]. Akshay et al. presented the Mobile Vocational Education (MoVE) units, which were designed to provide vocational training to rural and tribal populations of India. The system provides various levels of training for multiple vocations. Due to the software's portability and mobility and its low cost, it can provide training to a larger population than through traditional means [24]. Similarly, Muller and Ferreira introduced the Mechatronics: Access to Remote and Virtual E-Learning (MARVEL) product, focusing on expanding access to mechatronics vocational training [25]. MARVEL uses real and virtual environments and strives to create social contexts to provide a better learning experience. Oguzer et al. argue that, in order to improve vocational training education in Nigeria, access to computers and Information and Communication Technology (ICT) is vital [26]. While the cost associated with the development of a Virtual Reality-based training software could be expensive, the benefits that come from the increased education of a larger workforce far outweigh the cost of the associated technology. Our VR system would provide access in a similar way: VR headsets loaded with training scenarios can be repeated by many trainees to eventually provide a low-cost learning environment.

\subsection{Storytelling-Based Training}

Storytelling has been linked to increased memory retention [27,28]. Sarica and Usluel studied grade school students over 14 weeks to determine whether Digital Storytelling (DST) had an effect on writing and visual memory capacity [27]. They concluded, after significant results, that DST increased the children's cognitive capabilities in a shorter amount of time than other traditional methods. Gallets et al. presented evidence supporting this as well [28]. Groups of children that were presented information that was told in an interesting way by a storyteller comprehended and remembered the material more easily than if they were simply read to. The authors concluded that this was likely due to the engagement of the children, which allowed them to retain and understand the content. Storytelling has also made its way into the processes of vocational training and has had positive results. Andrews et al. provided an overview of various storytelling methods and their use in professional instruction [29]. They proposed that the brain's pattern recognition is the primary reason that storytelling-based instruction is effective. Magerko et al. proposed a way to create story-based training through the analysis of the trainee's current skill set and experience through the Interactive Storytelling Architecture for Training (ISAT) [30]. By placing emphasis on the skills that need the most work and creating an engaging environment, they argued that the training process will take less time [30]. Gandelman and Santoro proposed the use of group storytelling as a replacement for traditional training methods due to cost, time and content restrictions and offered a technique for its design [31]. Ladeira et al. [32] created a series of videos to teach bed-making and vacuuming skills to domestic workers in India. They found that a high motivational narrative along with a strong compelling action-a story hook-was key to improving the learning outcome. In later sections, we show how we build upon their work and construct a fictional narrative for vocational training.

\subsection{Combining VR and Storytelling}

Carlson et al. used a burr puzzle to test VR's effect on skill acquisition [33]. They found that by having color cues in Virtual Reality and the same colors in the physical world, the long-term skill retention was higher than when learning the burr puzzle in a purely physical environment. Jou and Wang also found success in training students within the Virtual Reality Learning Environment (VRLE) [34]. They emphasize the importance of practical training and highlight the benefits of the VRLE's ability to provide this training. VR has also been used to implement storytelling. Mollet and Arnaldi discuss the Giat Virtual Training (GVT) platform, a maintenance virtual reality training program that uses storytelling to train employees to 
develop certain skills [35]. The GVT platform is meant to be used as a trainer's tool, not as a replacement for an expert. They focus on creating scenarios based on constraints and goals, rather than a sequence of linear events. Lugrin et al. discuss storytelling in VR from an entertainment point of view [36]. They developed an immersive interactive narrative in which the user participates. The participants showed a willingness to engage in interactions and were active within the narrative in a productive way.

Wood and Reiners highlight the difficulties of traditional learning methods and the benefits of using virtual environments with storytelling elements [37]. They use the project nDiVE to show how storytelling and gamification can increase engagement and self-directed learning. Repeated use of the same virtual scenario can provide a trainee with various benefits, trade-offs or consequences of their actions. Gelsomini et al. [38] evaluated the use of storytelling and VR to provide therapies for children with Intellectual and developmental disorders and found that the system was beneficial in triggering a learning process and helped them stay focused in performing a task. Here, the storytelling content had positive effects on learning outcomes inside a VR environment on this group of users. Our vIS system utilizes storytelling inside of a virtual workplace to provide a cost-effective and controlled learning environment, while still providing the desired on-the-job context. It combines many aspects of the systems discussed here to provide a more complete learning experience focused on user engagement and knowledge retention. Rather than a tool, our system uses a complete scenario to provide instruction, demonstration and experience, without needing an expert to be present.

\section{Materials and Methods}

\subsection{System Design}

Our vIS system bridges the gap between Virtual Reality and storytelling by using an immersive virtual workplace environment and employing a creative, non-interactive, fictional story to provide vocational training. In this section, we give an overview of the skill training being provided and then explain the two main components of our system - the story and the virtual workplace environment—and provide the design decisions that led to the creation of our immersive story.

The system was implemented in Unity 3D with C\# scripting, and animations were done in Cinema4D. We used an HTC Vive head-mounted display (HMD) for our training simulation.

\subsection{Training Apparatus}

We designed our system to train users to use a mechanical micrometer; specifically, an outside micrometer. A micrometer is a measuring instrument that is used to make extremely precise measurements of objects and can measure within one one-thousandth on an inch. Such tools are especially important in manufacturing environments where measurements of objects such as pipes, valves or fittings need to be extremely precise to avoid potentially dangerous outcomes. An outside micrometer is designed to measure the thickness or outside diameter of a small object. Figure 1 shows the 3D model of the outside micrometer used in our study. 


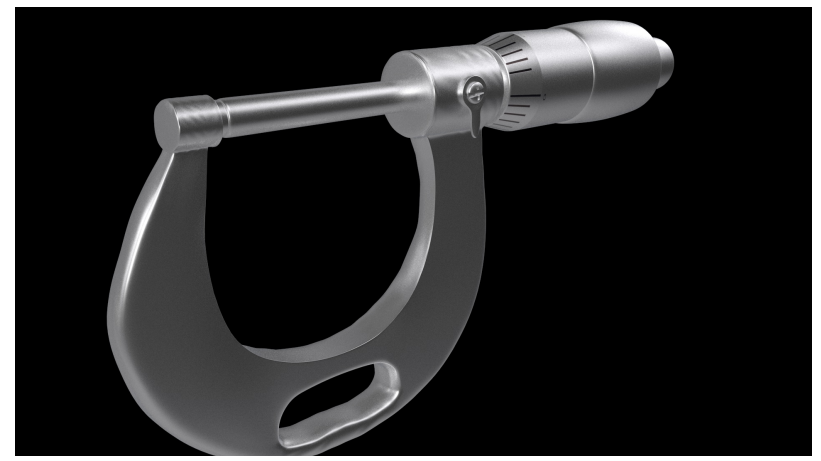

Figure 1. Three-dimensional model of an outside mechanical micrometer.

\subsection{Storytelling Content}

Storytellers aim to create content that is culturally and personally interesting and tap into human desires for adventure, fear, arousal, drama, love and hate. To create immersive stories, authors not only need to utilize traditional narrative strategies-such as creating compelling characters, twisting the story plots, adding story arcs, etc. - but they also need to aid the viewer's perceptual experience with immersive interfaces. Keeping these principles in mind, we build upon the work of Ladeira et al. [32] by embedding sequential instructions of how to use a micrometer into a narrative structure so that the instructions are preceded by the story plot and the skill training takes place during a story's character development phase.

Story Plot

The story begins outside of a manufacturing plant's workplace. David, a worker, is shown walking into the plant from the entrance. The floor of the plant is busy, with conveyor belts moving and workers walking past David and exchanging greetings. The scene is shown from a third-person perspective. David stops to look across the plan from left to right, looking for Remy. He spots Remy in the far-right corner of the building. Remy, our story's protagonist, is a plumber and is sitting at his desk. David walks up to Remy's desk; the sound of workers talking and the conveyor belt moving can be heard in the background. David stops at Remy's desk and begins chatting with him. A few moments later, a major pipe bursts behind Remy's desk, causing water leakage. Remy rushes to stop the water from leaking by affixing a temporary lid. David and Remy discuss the situation; they know that the lid is not going to hold in place for long, and so Remy rushes to find a replacement pipe to fix the leak. Remy tells David that he needs to cut a cylindrical cap to exactly the size of the leak and tells David that he is willing to teach him how to use a micrometer so that he can fix this problem in the future in case he is not in the office. The camera zooms in on the table on which Remy is setting up the anvil to demonstrate how to use the micrometer. Remy walks David through the entire procedure of how to use a micrometer on a cylindrical pipe, and when he finishes, he cuts the pipe to the right size and fixes the leaking pipe. David and Remy celebrate when the leak stops, ending the story on a higher note.

\subsection{Virtual Environment}

Our virtual environment is based on a manufacturing plant's workplace. The environment has an active conveyor belt and multiple workstations spread across the plant, with many workers actively working and walking around. The two main characters in the environment are David, who is a factory worker, and Remy, a plumber and our story's protagonist. Our virtual environment is not cluttered with all the components of a typical manufacturing plant, as we wanted to avoid causing any distraction to the viewer during the story runtime. The crux of the story plot occurs around Remy's desk. An anvil and a 
mechanical micrometer can be seen positioned at his desk; behind his desk is a water pipe which forms the center of our story's hook. All the characters in our environment have very lifelike features, and their movements and expressions very closely resemble those of an actual human being, keeping the entire experience as immersive as possible. The animations of our characters were done in Cinema4D and then imported into our virtual environment in Unity 3D.

\subsection{Design Decisions}

A large number of design decisions were taken to create a suitable fictional story. We started with story-boarding and made multiple iterations of the story's design to create a story that delivered the training information in the correct way and also elicited the right emotions from the user to aid in the long-term recall of the training content. Figure 2 shows the key scenes of our final storyboard.


Figure 2. Storyboard of our immersive virtual storytelling training system.

Design Decision \#1-Story format: The format of a story plays a huge role in how well the viewer remains connected to and engaged with the story content. In order to keep the story length short and avoid adding complications, we chose the most common form of narrative fiction-a three-act structure which divides the story into three parts:

1. Setup: This is used for exposition - the characters, the protagonist, their relationships, the environment setting and all relevant background information are laid out to the viewer.

2. Confrontation: This is usually triggered by an onset of an unexpected event, called a complicating action, followed by which the protagonist attempts to resolve the problem by learning new skills, often referred to as character development.

3. Resolution: Part of the story that features the resolution, preceded by the climax of the story.

Design Decision \#2-The complicating action: Commonly referred to as the story's hook, it was critical for us to pick the right event to elicit the right emotion from the viewer. Emotions play a huge role in learning [39], and eliciting the right emotions helps participants to remember the events following the complicating action for a longer time. Given that our story background was a virtual manufacturing plant's workplace and the task at hand was to train users on how to use a micrometer, we considered multiple complicating actions and tested them using empathy maps-a key tool used in the Design Thinking Process. We decided on a major pipe burst event causing water leakage as the complicating action to disrupt the flow of the story. 
Design Decision \#3-Visual perspective: In virtual environments, the two main types of perspectives available are first-person perspective (1PP), in which the camera is in the viewer's eye, and third-person perspective (3PP), in which the camera is at an adjustable distance and angle of view. The decision to choose between 1PP and 3PP was a crucial one. We iterated over multiple versions of the story and tested different combinations of perspectives: 1PP, 3PP and a combination of $1 \mathrm{PP}$ and 3PP. We found that 3PP worked well for our immersive story, and prior research comparing 1PP and 3PP for VR-based training [40] also confirmed that users prefer 3PP as it makes it easy to perform spatial navigation in the virtual environment. When testing the 3PP design prototype, users said it gave them a sense of control and safety when they were introduced to a new environment.

Design Decision \#4-Camera movement: We designed the camera movement in such a way that it allowed the viewer to easily focus on the main components of the story but also gave them the freedom to look around the surrounding environment in the viewer's field of vision through head movement only. The viewer could not move within the environment. As Virtual Reality is highly immersive and has a $360^{\circ}$ view, it was important to design an experience that focused the viewer's attention to the main activities in the story. Instead of deciding on only one type of camera movement, we decided to use a combination of two different camera movements. At the beginning of the story, the viewer had control over the camera movement as the character was slowly entering the factory, giving the viewer the ability to move their head and be able to see the overview of the environment. At the time of training, the free head movement was extremely limited, thus ensuring that the viewer pays attention to the micrometer training and is not distracted by other elements in the environment.

The speed of the camera movement during the story flow was determined, and the resulting video was at $35 \mathrm{fps}$ after multiple iterations of testing with users to avoid causing any unease to the user.

Design Decision \#5-Sound: An immersive story is less impactful without good sound design. Sound plays a huge role in how the user interacts and engages with the story and consumes the presented information. Salselas et al. [41] reviewed the literature on the role of sound in immersive storytelling and found that a good sound design modulates the attention of a viewer in a way that allows for an immersive user experience and allows the viewer to deduce that a narrative is being followed. We added sound for the factory environment-workers talking and working with heavy equipment—and when the main characters were engaged in a discussion, we reduced the background noise to move the focus to the character dialogues.

Design Decision \#6-Embedding training material: Adding the training material regarding the use of a micrometer in our story was critical to allow for long-term recall. We wanted to avoid creating our story in a way that would make it look as if it were simply an enhanced instructional video or presenting an overly informal storyline in which the viewer would not focus on the training material. To strike the right balance, we were aided by a few specialized vocational training instructors who helped us to craft our story dialogues in such a way that the viewer could connect with the main characters in the story. In our story, the main character talks aloud regarding how he plans to fix the broken pipe and walks the viewer through the various steps of using a micrometer and calculating the width of the pipe needed to fix the leakage.

\subsection{User Study Design}

To test the effectiveness of our system, we conducted a training and recall study to answer the following research questions:

1. R1: Can users effectively use our vIS system to learn a new skill-in our case, learning how to use a micrometer-and be able to apply it immediately after training? 
2. R2: Can users of our vIS system recall the learning a week after the training?

3. R3: Is the overall effectiveness and usability of our vIS system better in comparison to traditional methods of training; i.e., text-based manuals and 2D video training?

We also wanted to determine the usability of our vIS system to inform future design decisions, and as a result, a system usability scale (SUS) questionnaire [20] was administered to all the participants.

\subsection{Study Design}

In order to empirically evaluate the above research questions, we developed a between-subject experiment in which participants were allocated, in a round-robin format, to one of three training methods:

1. vIS: In this method, the participants were given HTC Vive headsets and a headphone to view the immersive story. The immersive story was $2 \mathrm{~min}$ and $50 \mathrm{~s}$ long.

2. Two-dimensional video: In this method, participants were presented with a 2D tutorial video, without any storytelling components, which explained all the steps of using a micrometer. The video was 3 min long.

3. Text manual: In this method—-the traditional approach to learning a new skill—participants were given an instructional manual which outlined all the steps of using a micrometer. The manual contained pictures of the components of a micrometer along with the usage steps. The length of the manual was 2 pages. No storytelling elements were included in this manual.

In all of the above methods, the participants were given the freedom to repeat the training again until they felt confident in the use of a micrometer. To measure the learning and recall of these methods, our user study was divided into two phases with a gap of seven days between both phases.

1. Day 1: Training session. Participants were assigned to one of the above-mentioned training methods, and they interacted with the assigned system until they felt confident in the use of a micrometer. Once the training was complete, they were given a cylindrical object and a micrometer and were asked to measure the diameter of the object using the same steps taught in the training material. Finally, their answers along with the SUS scale responses were then collected.

2. Day 7: Recall session (seven days after the training session). Participants were asked to calculate the diameter of the same cylindrical object, and their answers were collected.

The study was conducted on a university campus, in a controlled lab environment, and was approved by the University of Texas Arlington Institutional Review Board (UTA IRB, 2020-0055). We recruited participants via word of mouth. During the study, only one participant indicated having some knowledge on the use of a micrometer.

\subsection{Study Procedure}

\subsubsection{Training Session}

Participants were presented with an Informed consent form. After giving consent, they were assigned to one of the three training methods in a round-robin style and were told the aim of the study. They were given the freedom to repeat the training any number of times until they felt confident in the use of a micrometer. At the end of the training, they were given a round cylindrical object and were asked to measure the diameter of the object. They were then given an online form in which they had to submit their answer along with filling out a questionnaire to capture the SUS scores. 


\subsubsection{Recall Session}

Participants returned for a follow-up study after seven days of completing their training and were presented with a different cylindrical object. They were asked to calculate the diameter of the object and submit the answer in our online form.

\subsection{Data Collection}

To evaluate the usability and effectiveness of our system, we collected the following types of information throughout this study:

- Demographic information of all the participants;

- The number of times each participant repeated their training in the training session;

- Total time taken by each participant to complete the training;

- Total time taken by each participant to calculate the diameter of the object post-training in the training session;

- Accuracy of their calculations in the training session;

- Responses to our SUS questionnaire post-training;

- Total time taken by each participant to calculate the diameter of a different object in the recall session;

- Accuracy of their calculations in the recall session.

\section{Results}

As stated above, the purpose of the usability study was to assess the effectiveness and long-term recall rate of vIS and compare it against the traditional methods of training. We conducted a one-way analysis of variance (ANOVA) to test for statistically significant differences between the three different training methods and then conducted the Tukey post-hoc test to reveal the level of significance. Wherever possible, we also presented the analyzed data using box-and-whisker plot diagrams.

\subsection{Demographics}

Thirty adults participated in our usability study. Most were students at our university from various degree programs; none of them had any prior experience with vocational training, and only one of them identified having some prior knowledge of the use of a micrometer, but they were not confident in recalling the steps when asked. Among those who reported their age, the age range varied between 19 to 45, with a mean age of 25.85 . In total, $70 \%$ of participants identified their gender as male and $30 \%$ identified as female.

\subsection{Training Time}

We measured the training time as the total time taken by participants to complete the training session. Since participants were allowed to view the training material any number of times until they felt confident in the use of a real micrometer, this time included the time taken for all their attempts to view the training. This time was calculated manually by the usability study invigilator using a stopwatch. Table 1 summarizes the training time for all three training methods.

There was a statistically significant difference in the training time between the three training methods, as determined by one-way ANOVA $(\mathrm{F}(2,27)=11.65914, p=0.000224)$. Furthermore, the Tukey post-hoc test revealed that training time was statistically significantly lower in vIS compared to the text-based manual method, at $p<0.05$. There was no statistically significant difference between our vIS method and the $2 \mathrm{D}$ video training method. 
Table 1. Training time of all three training methods (in seconds).

\begin{tabular}{cccc}
\hline Training Method & Mean & Median & Std Dev \\
\hline vIS & 289 & 340 & 82.11 \\
2D video & 196.8 & 197 & 24.86 \\
Text manual & 455.9 & 367 & 192.40 \\
\hline
\end{tabular}

\subsection{Post-Training Measurement Time}

We measured this as the time taken by the participant to calculate the diameter of a cylindrical object using a micrometer immediately after completing the training session. This time was calculated manually by the usability study invigilator using a stopwatch. Table 2 summarizes the measurement time comparison of all three training methods.

We observed no statistically significant difference in the measurement time for all three training groups.

Table 2. Post-training measurement time comparison of all three training methods (in seconds).

\begin{tabular}{cccc}
\hline Training Method & Mean & Median & Std Dev \\
\hline vIS & 140.128 & 126.23 & 31.50 \\
2D video & 143.339 & 129.87 & 34.89 \\
Text manual & 177.248 & 164.68 & 74.73 \\
\hline
\end{tabular}

\subsection{Recall Measurement Time}

We measured this time as the time taken by the participant to calculate the diameter of the same cylindrical object during the recall session, exactly 1 week after their training session. This time was calculated manually by the usability study invigilator using a stopwatch. Table 3 summarizes the recall measurement time comparison of all three training methods.

In contrast to the post-training measurement time, we observed a statistically significant difference in the one-way ANOVA test $(\mathrm{F}(2,27)=3.86, p=0.033542)$. The Tukey post-hoc test revealed a statistically significant difference of $p<0.05$ between the vIS and text manual training methods and no statistical significance between the vIS and 2D video methods.

Table 3. Recall measurement time comparison of all three training methods (in seconds).

\begin{tabular}{cccc}
\hline Training Method & Mean & Median & Std Dev \\
\hline vIS & 209.5 & 205 & 14.01 \\
2D video & 213 & 213 & 11.16 \\
Text manual & 223.9 & 221 & 10.81 \\
\hline
\end{tabular}

\subsection{Training vs. Recall Measurement Accuracy}

The accuracy of the measurements was calculated using the formula:

$$
\frac{\left|V_{a}-V_{o}\right|}{V_{a}} \times 100
$$

where $V_{a}$ is the acceptable value of the cylindrical object and $V_{o}$ is the measured value of the cylindrical object. Before starting the usability study, we calculated the acceptable value of the cylindrical object by performing 10 measurements and taking the average of these calculations to the nearest thousandth of an 
inch. We then compared the participants' measurements against our acceptable value and calculated each participant's accuracy percentage for both sessions.

Figure 3 shows the comparison of measurement accuracy during the training and recall sessions. While no statistically significant difference was observed in the accuracy during the training session, we observed a significant difference in the recall session $(\mathrm{F}(2,27)=2.54, p=0.047554)$. The Tukey post-hoc test revealed a statistically significant difference of $p<0.05$ between the vIS and 2D video methods and the vIS and text manual methods.

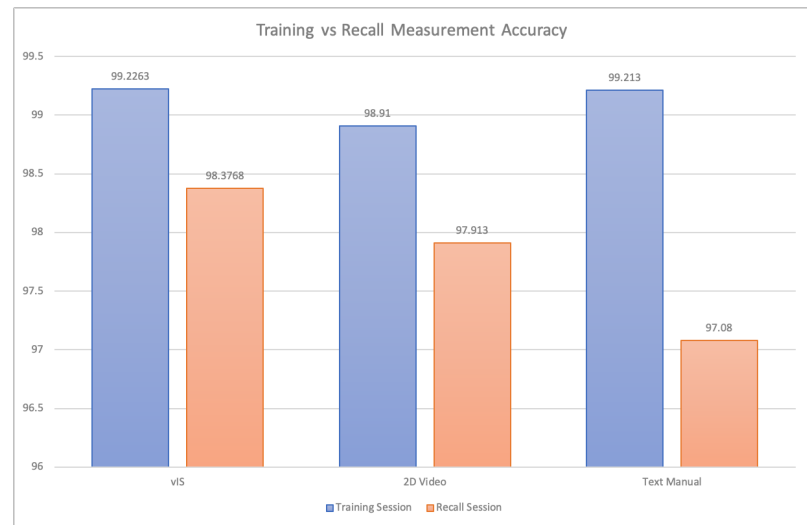

Figure 3. Training vs. Recall Measurement Accuracy.

\subsection{SUS Survey}

In our survey, we asked the participants to answer the system usability questions on a five-point Likert-scale (1: Strongly Disagree, 5: Strongly Agree). Overall, the average SUS scores for the vIS, 2D video and text manual methods were 76, 72 and 62, respectively. A SUS score of 68 and above was considered to be above average. These scores show that, while participants in the text manual group certainly did not find the method to be usable, participants in the vIS method group perceived the system's usability to be higher than that of the 2D video method.

\section{Discussion}

Virtual Reality training systems are now starting to become commercially available. However, the combination of storytelling narratives with an immersive training system has been largely unexplored. Our vIS system explores a promising new direction to improving the long-term memorability of training material by embedding the training instructions inside a fictional narrative. This is a pioneering and unique Virtual Reality-based system that compares extremely well with the traditional learning methods.

Considering the training session time, we can see that the text manual method represented the longest training sessions, whereas the 2D video method had the shortest length. While the vIS group took roughly a minute and a half longer than the 2D video method, it is worth noting that the storytelling system had the instructions embedded inside a story; thus, it was expected that this group would require slightly longer than those watching a $2 \mathrm{D}$ video. While there is no a great level of statistical significance in the difference between our storytelling system and the 2D video, vIS clearly outperformed the text manual method of learning. This is in line with the expectations of previous work, such as that by Chao et al. [42] in which the VR training was considered the best in comparison to technical manuals.

The results of the post-training measurement task showcased the ability of humans to recall instructions from their short-term memory. As described by Atkinson et al. [43], theretrieval of information from our short-term memory store is quite fast; thus, we see a nearly ideal and equal measurement time 
resulting from our storytelling system and $2 \mathrm{D}$ video method. The result of a very high measurement time for the text manual method was surprising to us, as we expected the time to be nearly equal to the other methods.

It is interesting to see the long-term memory recall result in the measurement time during the recall phase, as measurement times increased after a gap of seven days, which was clearly a sign of the complication of retrieving information from our long-term memory store, as described by Atkinson et al. [43]. We see that the measurement time of participants from the vIS group was the shortest of all the methods, proving the power of storytelling in aiding in the long-term recall of training instructions. We expected the measurement time of the 2D video group to be less than that of the text manual group, as video-based training has been proven to be a stronger tool for encoding information for long term retention.

In combination with the measurement time observed during the recall phase, the significance of our storytelling system's capability to provide long-term training can also be found by comparing the accuracy of measurements in training and recall sessions. As evident from Figure 3, although the accuracy reduced for all methods in the recall phase, the accuracy of measurements remained consistently higher for the vIS system compared to the traditional methods. A statistical difference of $p<0.05$ proves that our immersive storytelling system performed extremely well regarding the long-term retention of training material.

These results demonstrate that, while our training system fares equally in comparison to traditional training methods, the benefits of adding the storytelling content appear most in the long-term recall of the training content. Although vIS users had statistically insignificantly different recall times compared to other methods, we place more weight on the combination of recall time and measurement accuracy in the recall session, because the pair of these variables is what defines a usable training system. vIS performed better than other methods with regards to the combination of these two variables. Clearly, vIS has a large degree of usability potential, as shown in the post-survey questionnaires pointing to a higher-than-average SUS score. Further evaluation of our system is needed with a larger number of participants in order to verify our findings.

\section{Conclusions}

To increase the skill-level of a country's population, the need for a cost-effective vocational training solution has risen in the last few years. While there are several Virtual Reality-based training systems, the field of immersive storytelling-based training systems has largely been unexplored. In this paper, we presented vIS, a pioneering Virtual Reality-based immersive storytelling system that employs storytelling as a medium to impart training to users. Our system focuses on teaching users how to use an industrial micrometer. We conducted a user study with 30 participants to evaluate the system's effectiveness in providing long-term training and compared it against traditional forms of training: 2D video and text-based methods. Our results show that the use of storytelling inside a virtual environment is very effective compared to text-based training and equally effective as $2 \mathrm{D}$ video-based training; furthermore, our approach is more engaging to users than $2 \mathrm{D}$ video and text-based techniques.

vIS is the first step towards the use of storytelling for immersive training; thus, we only compared its effectiveness against the traditional forms of training and required the user to practice with a micrometer outside the virtual environment. In the next step, we intend to add interaction with a micrometer inside the virtual environment and bridge the interaction with the ongoing story to make it a seamless immersive experience. Our far-reaching goal is to enrich our vocational training system with an intelligent story in an immersive environment in which users can interact with the environment and be able to be trained on-demand without the need for in-person training. 
Author Contributions: S.D. conceived and designed the study and wrote the paper. L.O. was responsible for the development of the VR platform. C.W. contributed to the manuscript preparation. S.D. and F.M. supervised the study and managed the project. F.M. provided her expertise in vocational training. All authors have read and agreed to the published version of the manuscript.

Funding: This research was funded by the National Science Foundation (NSF) under award numbers NSF-PFI 1719031.

Acknowledgments: The authors would like to thank all participants of the user study, and to Maria Kyrarini for proof-reading the manuscript.

Conflicts of Interest: The authors declare no conflict of interest.

\section{References}

1. Biavaschi, C.; Eichhorst, W.; Giulietti, C.; Kendzia, M.J.; Muravyev, A.; Pieters, J.; Rodríguez-Planas, N.; Schmidl, R.; Zimmermann, K.F. Youth Unemployment and Vocational Training; IZA Discussion Paper No. 6890; Institute of Labor Economics (IZA): Bonn, Germany, 2012; Available online: https:/ / ssrn.com/abstract=2158300 (accessed on 20 October 2020).

2. Chakravarty, S.; Lundberg, M.; Nikolov, P.; Zenker, J. Vocational training programs and youth labor market outcomes: Evidence from Nepal. J. Dev. Econ. 2019, 136, 71-110. [CrossRef]

3. Knoke, D.; Kalleberg, A.L. Job training in US organizations. Am. Sociol. Rev. 1994, 59, 537-546. [CrossRef]

4. Tsiakas, K.; Abujelala, M.; Lioulemes, A.; Makedon, F. An intelligent interactive learning and adaptation framework for robot-based vocational training. In Proceedings of the 2016 IEEE Symposium Series on Computational Intelligence (SSCI), Athens, Greece, 6-9 December 2016; IEEE: New York, NY, USA, 2016; pp. 1-6.

5. Tsiakas, K.; Papakostas, M.; Theofanidis, M.; Bell, M.; Mihalcea, R.; Wang, S.; Burzo, M.; Makedon, F. An interactive multisensing framework for personalized human robot collaboration and assistive training using reinforcement learning. In Proceedings of the 10th International Conference on PErvasive Technologies Related to Assistive Environments, Island of Rhodes, Greece, 21-23 June 2017; pp. 423-427.

6. Tsiakas, K.; Abujelala, M.; Makedon, F. Task engagement as personalization feedback for socially-assistive robots and cognitive training. Technologies 2018, 6, 49. [CrossRef]

7. Abujelala, M.; Gupta, S.; Makedon, F. A Collaborative Assembly Task to Assess Worker Skills in Robot Manufacturing Environments. In Proceedings of the 11th PErvasive Technologies Related to Assistive Environments Conference, PETRA '18, Corfu, Greece, 26-29 June 2018; Association for Computing Machinery: New York, NY, USA, 2018; pp. 118-119, [CrossRef]

8. Schmidt, M.W.; Kowalewski, K.F.; Schmidt, M.L.; Wennberg, E.; Garrow, C.R.; Paik, S.; Benner, L.; Schijven, M.P.; Müller-Stich, B.P.; Nickel, F. The Heidelberg VR Score: Development and validation of a composite score for laparoscopic virtual reality training. Surg. Endosc. 2019, 33, 2093-2103. [CrossRef] [PubMed]

9. Roldán, J.J.; Crespo, E.; Martín-Barrio, A.; Peña-Tapia, E.; Barrientos, A. A training system for Industry 4.0 operators in complex assemblies based on virtual reality and process mining. Robot. Comput. Integr. Manuf. 2019, 59, 305-316. [CrossRef]

10. Johnson, C.M.; Vorderstrasse, A.A.; Shaw, R. Virtual worlds in health care higher education. J. Virtual Worlds Res. 2009, 2. [CrossRef]

11. Ryan, E.; Poole, C. Impact of Virtual Learning Environment on Students' Satisfaction, Engagement, Recall, and Retention. J. Med. Imaging Radiat. Sci. 2019, 50, 408-415. [CrossRef] [PubMed]

12. Mount, N.; Chambers, C.; Weaver, D.; Priestnall, G. Learner immersion engagement in the 3D virtual world: principles emerging from the DELVE project. Innov. Teach. Learn. Inf. Comput. Sci. 2009, 8, 40-55. [CrossRef]

13. Sauzéon, H.; Pala, P.A.; Larrue, F.; Wallet, G.; Déjos, M.; Zheng, X.; Guitton, P.; N’Kaoua, B. The use of virtual reality for episodic memory assessment. Exp. Psychol. 2012, 59, 99-108.

14. Reggente, N.; Essoe, J.K.; Baek, H.Y.; Rissman, J. The method of loci in virtual reality: explicit binding of objects to spatial contexts enhances subsequent memory recall. J. Cogn. Enhanc. 2020, 4, 12-30. [CrossRef] 
15. Krokos, E.; Plaisant, C.; Varshney, A. Virtual memory palaces: Immersion aids recall. Virtual Real. 2019, $23,1-15$. [CrossRef]

16. Ebert, D.; Gupta, S.; Makedon, F. Ogma: A virtual reality language acquisition system. In Proceedings of the 9th ACM International Conference on Pervasive Technologies Related to Assistive Environments, Corfu, Greece, 29 June-1 July 2016; pp. 1-5.

17. Smeda, N.; Dakich, E.; Sharda, N. The effectiveness of digital storytelling in the classrooms: A comprehensive study. Smart Learn. Environ. 2014, 1, 6. [CrossRef]

18. Robin, B.R. Digital storytelling: A powerful technology tool for the 21st century classroom. Theory Pract. 2008, 47, 220-228. [CrossRef]

19. Jack, H. State of Manufacturing Education. In Proceedings of the 2013 ASEE Conference, Atlanta, GA, USA, 23-26 June 2013.

20. Brooke, J. SUS-A quick and dirty usability scale. Usability Eval. Ind. 1996, 189, 4-7.

21. Billett, S. Workplace learning: its potential and limitations. Educ. Train. 1995, 37, 20-27. [CrossRef]

22. Smith, P.J. Workplace learning and flexible delivery. Rev. Educ. Res. 2003, 73, 53-88. [CrossRef]

23. De Bruijn, E.; Leeman, Y. Authentic and self-directed learning in vocational education: Challenges to vocational educators. Teach. Teach. Educ. 2011, 27, 694-702. [CrossRef]

24. Akshay, N.; Sreeram, K.; Anand, A.; Venkataraman, R.; Bhavani, R.R. MoVE: Mobile vocational education for rural India. In 2012 IEEE International Conference on Technology Enhanced Education (ICTEE), Kerala, India, 3-5 January 2012; IEEE: New York, NY, USA, 2012; pp. 1-5.

25. Mueller, D.; Ferreira, J.M. MARVEL: A mixed-reality learning environment for vocational training in mechatronics. In Proceedings of the Technology Enhanced Learning International Conference (TEL 03), Milan, Italy, 20-21 November 2003.

26. Oguzor, N.S. computer usage as instructional resources for vocational training in Nigeria. Educ. Res. Rev. 2011, 6, 395-402. [CrossRef]

27. Sarıca, H.Ç.; Usluel, Y.K. The effect of digital storytelling on visual memory and writing skills. Comput. Educ. 2016, 94, 298-309. [CrossRef]

28. Gallets, M.P. Storytelling and Story Reading: A Comparison of Effects on Children's Memory and Story Comprehension. Master's Thesis, East Tennessee State University, Johnson City, TN, USA, 2005.

29. Andrews, D.H.; Hull, T.D.; Donahue, J.A. Storytelling as an Instructional Method: Descriptions and Research Questions; Technical Report; Oak Ridge Inst for Science and Education Tn: Oak Ridge, TN, USA, 2009.

30. Magerko, B.; Wray, R.; Holt, L.; Stensrud, B. Improving interactive training through individualized content and increased engagement. In Proceedings of the Interservice/Industry Training, Simulation \& Education Conference (I/ITSEC), Orlando, FL, USA, 6-9 December 2005; pp. 1-11.

31. Gandelman, R.T.; Santoro, F.M. Group Storytelling in Organizational Training. In Proceedings of the IADIS International Conference on Information System, Porto, Portugal, 18-20 March 2010; pp. 315-322.

32. Ladeira, I.; Cutrell, E. Teaching with storytelling: An investigation of narrative videos for skills training. In Proceedings of the 4th ACM/IEEE International Conference on Information and Communication Technologies and Development, London, UK, 13-16 December 2010; pp. 1-10.

33. Carlson, P.; Peters, A.; Gilbert, S.B.; Vance, J.M.; Luse, A. Virtual training: Learning transfer of assembly tasks. IEEE Trans. Vis. Comput. Graph. 2015, 21, 770-782. [CrossRef]

34. Jou, M.; Wang, J. Investigation of effects of virtual reality environments on learning performance of technical skills. Comput. Hum. Behav. 2013, 29, 433-438. [CrossRef]

35. Mollet, N.; Arnaldi, B. Storytelling in virtual reality for training. In International Conference on Technologies for E-Learning and Digital Entertainment; Springer: Berlin, Germany, 2006; pp. 334-347.

36. Lugrin, J.L.; Cavazza, M.; Pizzi, D.; Vogt, T.; André, E. Exploring the usability of immersive interactive storytelling. In Proceedings of the 17th ACM Symposium on Virtual Reality Software and Technology, Hong Kong, China, 22-24 November 2010; pp. 103-110.

37. Wood, L.C.; Reiners, T. Storytelling to immersive learners in an authentic virtual training environment. In Gamification in Education and Business; Springer: Berlin, Germany, 2015; pp. 315-329. 
38. Gelsomini, M.; Garzotto, F.; Montesano, D.; Occhiuto, D. Wildcard: A wearable virtual reality storytelling tool for children with intellectual developmental disability. In Proceedings of the 2016 38th Annual International Conference of the IEEE Engineering in Medicine and Biology Society (EMBC), Orlando, FL, USA, 16-20 August 2016; IEEE: New York, NY, USA, 2016; pp. 5188-5191.

39. LeDoux, J.E. Brain mechanisms of emotion and emotional learning. Curr. Opin. Neurobiol. 1992, 2, $191-197$. [CrossRef]

40. Salamin, P.; Tadi, T.; Blanke, O.; Vexo, F.; Thalmann, D. Quantifying effects of exposure to the third and first-person perspectives in virtual-reality-based training. IEEE Trans. Learn. Technol. 2010, 3, 272-276. [CrossRef]

41. Salselas, I.; Penha, R. The role of sound in inducing storytelling in immersive environments. In Proceedings of the 14th International Audio Mostly Conference: A Journey in Sound, Nottingham, UK, 18-20 September 2019; pp. 191-198.

42. Chao, C.J.; Wu, S.Y.; Yau, Y.J.; Feng, W.Y.; Tseng, F.Y. Effects of three-dimensional virtual reality and traditional training methods on mental workload and training performance. Hum. Factors Ergon. Manuf. Serv. Ind. 2017, 27, 187-196. [CrossRef]

43. Atkinson, R.C.; Shiffrin, R.M. The control of short-term memory. Sci. Am. 1971, 225, 82-91. [CrossRef] [PubMed]

Publisher's Note: MDPI stays neutral with regard to jurisdictional claims in published maps and institutional affiliations. 